\title{
Pacific
}

Journal of

Mathematics

\section{THE WEIL REPRESENTATION AND GAUSS SUMS}

\section{ANTONIA WiLsON BLUHER}




\title{
THE WEIL REPRESENTATION AND GAUSS SUMS
}

\author{
ANtonia Bluher
}

We use the Weil representation to evaluate certain Gauss sums over a local field, up to \pm 1 . Also we construct a cocycle on $\operatorname{Sp}(2 m, \mathbb{R})$ with a simple formula on the maximal compact torus and we show how to lift homomorphisms $j: \mathrm{Sp}(2 n, \mathbb{R}) \rightarrow$ $\mathrm{Sp}(2 m, \mathbb{R})$ to the double covers of these groups.

\section{Introduction.}

Let $F$ be a self-dual local field of char $\neq 2$, for instance $F=\mathbb{R}, \mathbb{C}$, or a finite extension of $\mathbb{Q}_{p}$. For most of the paper we will assume $F \neq \mathbb{C}$. Let $\chi$ be a nontrivial additive character of $F$. Then all additive characters of $F$ have the form $\lambda \chi$ for some $\lambda \in F$, where $\lambda \chi(t)=\chi(\lambda t)$. We consider the following unitary operators on $L^{2}\left(F^{m}\right)$ :

$$
\begin{gathered}
(\mathbf{a}(A) \Phi)(X)=|\operatorname{det} A|_{F}^{1 / 2} \Phi(X A) \quad \text { for } A \in \mathrm{GL}_{m}(F), \\
\mathbf{n}(B) \Phi(X)=\chi\left(X B X^{T} / 2\right) \Phi(X) \quad \text { for } B=B^{T} \in \mathrm{M}_{m}(F)
\end{gathered}
$$

$$
\left(\mathcal{F}_{j} \Phi\right)(X)=\int_{F^{j}} \Phi\left(Y_{1}, \ldots, Y_{j}, X_{j+1}, \ldots, X_{m}\right) \chi\left(X_{1} Y_{1}+\cdots+X_{j} Y_{j}\right) d Y
$$

$$
(\iota(t) \Phi)(X)=t \Phi(X), \quad t \in \mathbf{T}=\{z \in \mathbb{C} \mid z \bar{z}=1\} .
$$

Here $\Phi$ is a nice function in $L^{2}\left(F^{m}\right)$ (to be precise, $\Phi$ belongs to the Schwartz space $\left.\mathcal{S}\left(F^{m}\right)\right), d Y$ is an additive Haar measure on $F^{j}$ normalized so that $\mathcal{F}_{j}^{2}=\mathbf{a}\left(\operatorname{diag}\left\{-I_{j}, I_{m-j}\right\}\right)$ for $0 \leq j \leq m$, and $|a|_{F}$ for $a \in F$ is the modulus function, determined by $d(y a)=|a|_{F} d y$ for a Haar measure $d y$ on $(F,+)$. All our vectors are row vectors. We will usually suppress the symbol $\iota$; that is, identify $t$ with $\iota(t)$ for $t \in \mathbf{T}$. Let $\mathrm{Mp}=\mathrm{Mp}\left(F^{m}\right)$ be the topological group generated by all the above operators. We call this the metaplectic group. This group is independent of $\chi$ since $\lambda \chi\left(X B X^{T} / 2\right)=\chi\left(X(\lambda B) X^{T} / 2\right)$ and $\mathbf{a}\left(\left({ }^{\lambda I_{j}}{ }^{I_{m-j}}\right)\right) \mathcal{F}_{j, \chi}=\mathcal{F}_{j, \lambda \chi}$, where we have added a subscript to the Fourier 
transform $\mathcal{F}_{j}$ for clarification. Let $\mathrm{Sp}=\mathrm{Sp}(2 m, F)$ denote the symplectic group consisting of all $2 m \times 2 m$ matrices $\tau$ such that $\tau^{T} w_{m} \tau=w_{m}$, where $w_{m}=\left(\begin{array}{cc}0 & I \\ -I & 0\end{array}\right), I=I_{m}$. This group is generated by the matrices $\{\underline{a}(A) \mid A \in$ $\left.\mathrm{GL}_{m}(F)\right\},\left\{\underline{n}(B) \mid B=B^{T} \in \mathrm{M}_{m}(F)\right\}$, and $w_{m}$, where

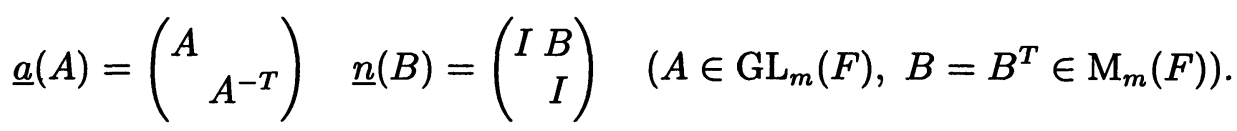

Theorem (Segal, Shale, Weil). There is a homomorphism $\pi=\pi_{\chi}: \mathrm{Mp} \rightarrow$ Sp such that

$$
\pi(\mathbf{a}(A))=\underline{a}(A), \quad \pi(\mathbf{n}(B))=\underline{n}(B), \quad \pi(\iota(t))=I_{2 m}, \quad \pi\left(\mathcal{F}_{j}\right)=w_{j},
$$

where

$$
w_{j}=\left(\begin{array}{cccc}
0_{j} & & & I_{j} \\
& I_{m-j} & 0_{m-j} \\
-I_{j} & & 0_{j} & \\
& 0_{m-j} & I_{m-j}
\end{array}\right) .
$$

Moreover, there is an exact sequence of topological groups

$$
1 \rightarrow \mathbf{T} \stackrel{\iota}{\longrightarrow} \mathrm{Mp} \stackrel{\pi}{\longrightarrow} \mathrm{Sp} \rightarrow 1 \text {. }
$$

If $F=\mathbb{C}$, this sequence splits. If $F \neq \mathbb{C}$ then $M p$ contains a subgroup $\widetilde{\mathrm{Sp}}=\widetilde{\mathrm{Sp}}(2 m, F)$ such that $\left.\pi\right|_{\widetilde{\mathrm{Sp}}}: \widetilde{\mathrm{Sp}} \rightarrow \mathrm{Sp}$ is a nontrivial two-fold cover of Sp.

From now on assume $F \neq \mathbb{C}$. The realization $\widetilde{\mathrm{Sp}} \subset U\left(L^{2}\left(F^{m}\right)\right)$ is known as the Weil, oscillator, or Segal-Weil-Shale representation. The group Mp and the projection $\pi$ have much better definitions in terms of a certain centralizing property these operators have with respect to a unitary representation of the Heisenberg group; see [R1, §3.2]. Note that

$$
\pi_{\lambda \chi}(\sigma)=\left(\begin{array}{ll}
I & \\
& \lambda I
\end{array}\right) \pi_{\chi}(\sigma)\left(\begin{array}{cc}
I & \\
& \lambda^{-1} I
\end{array}\right) \quad \text { for } \sigma \in \mathrm{Mp} .
$$

The main result of this paper is that a certain Gauss sum is computed up to \pm 1 . The idea of the proof is to compare two sections of the homomorphism $\pi$. If $F=\mathbb{R}$ or if $F$ is a nonarchimedean field whose residue characteristic is $\neq 2$ then there is a splitting homomorphism $\mathbf{k}: K \rightarrow \mathrm{Mp}$, where $K$ is a certain maximal compact subgroup of Sp. This section can be compared to the standard section $r_{0}: \mathrm{Sp} \rightarrow \mathrm{Mp}$ (see (4.1)) which was defined by Rao. Define $x_{0}: K \rightarrow \mathbf{T}$ by the formula $r_{0}(k)=x_{0}(k) \mathbf{k}(k)$. We will find 
an expression for $x_{0}(k)$ as a Gauss sum. The operators in $\widetilde{\mathrm{Sp}}$ are known explicitly, thus $y_{0}(g)$ and $y(k)$ may be found (Prop. 2 and Lemma 7) such that $y_{0}(g) r_{0}(g) \in \widetilde{\mathrm{Sp}}$ and $y(k) \mathbf{k}(k) \in \widetilde{\mathrm{Sp}}$ for each $g \in \mathrm{Sp}, k \in K$. Then $x_{0}(k) y_{0}(k) y(k)^{-1} \in \widetilde{\mathrm{Sp}} \cap \mathbf{T}=\{ \pm 1\}$. In this way the exact value of the Gauss sum $x_{0}(k)$ may be computed, up to \pm 1 . A technical difficulty is that one must find an explicit formula for the Bruhat decomposition on $K$ in order to compare the sections $r_{0}$ and $\mathbf{k}$; this is done in Lemma 9.

Now we state our result explicitly. Let $F=\mathbb{R}$ or $F=$ a self-dual nonarchimedean local field such that 2 is a unit in the ring $\mathfrak{o}_{F}$ of algebraic integers of $F$. Let $\chi(t)=e^{2 \pi i t}$ if $F=\mathbb{R}$, and let $\chi$ be any additive character such that $\mathfrak{a}_{\chi}=\mathfrak{a}_{F}$ if $F$ is nonarchimedean, where

$$
\mathfrak{a}_{\chi}=\left\{x \in F \mid \chi(x y)=1 \quad \text { for all } y \in \mathfrak{o}_{F}\right\} .
$$

For example, if $F=\mathbb{Q}_{p}$, one could take $\chi=\chi_{p}$ to be the unique additive character such that $\chi_{p}\left(a / p^{n}\right)=e^{-2 \pi i a / p^{n}}$ when $a, n \in \mathbb{Z}$, and if $F$ is a finite extension of $\mathbb{Q}_{p}$ one could take $\chi=\lambda \chi_{p} \circ \operatorname{tr}_{F / \mathbb{Q}_{p}}$, where $\lambda$ is a generator for the inverse different of $F$. Let $K=\operatorname{Sp}\left(2 m, \mathfrak{o}_{F}\right)$ if $F$ is nonarchimedean and

$$
K=\left\{\left(\begin{array}{cc}
\alpha & \beta \\
-\beta & \alpha
\end{array}\right) \mid(\alpha+i \beta)(\alpha-i \beta)^{T}=I_{m}\right\} \quad \text { if } F=\mathbb{R}
$$

Define $\Phi_{0} \in L^{2}\left(F^{m}\right)$ to be the characteristic function of $\mathfrak{o}_{F}^{m}$ if $F$ is nonarchimedean and $\Phi_{0}(X)=e^{-\pi X \cdot X}$ if $F=\mathbb{R}$. Let $k=\left(\begin{array}{ll}A & B \\ C & D\end{array}\right) \in K$ and $j=$ $\operatorname{rank}(C)$. It is not hard to see (Lemma 8) that there exist $\pi_{1}, \pi_{2} \in \mathrm{SO}(m, F)$ such that all entries of $\pi_{i}$ are 0,1 , or -1 and such that the top left $j \times j$ minor of $\pi_{1} C \pi_{2}$ is invertible. Let

$$
\pi_{1} C \pi_{2}=\left(\begin{array}{ll}
C_{1} & C_{2} \\
C_{3} & C_{4}
\end{array}\right), \quad \pi_{1} D \pi_{2}=\left(\begin{array}{cc}
D_{1} & D_{2} \\
D_{3} & D_{4}
\end{array}\right),
$$

where $C_{1}, D_{1}$ are $j \times j$ matrices and $C_{4}, D_{4}$ are $(m-j) \times(m-j)$ matrices. For $a, b \in F^{\times}$let $(a, b)_{F} \in\{ \pm 1\}$ denote the Hilbert symbol: $(a, b)_{F}=1$ or -1 according as $a$ is or is not a norm in $F(\sqrt{b})$. In particular, if $F=\mathbb{R}$ then $(a, b)_{\mathbb{R}}=-1$ iff $a$ and $b$ are both negative.

Main Theorem. Let $k \in K-P$ and $\pi_{1}, \pi_{2}, C_{1}, C_{2}, \ldots, D_{4}$ be as above. Put $\eta=\operatorname{det}\left(\begin{array}{cc}-C_{1} & D_{2} \\ -C_{3} & D_{4}\end{array}\right)$. Then $\eta \neq 0$, and the quantity

$$
x_{0}(k)=\left|\operatorname{det} C_{1}\right||\eta|^{-1 / 2} \int_{F^{j}} \chi\left(y\left(D_{1} C_{1}^{T}+D_{2} C_{2}^{T}\right) y^{T} / 2\right) \Phi_{0}\left(y C_{1}, y C_{2}\right) d y
$$

is independent of the choice of $\pi_{1}$ and $\pi_{2}$. Furthermore, $x_{0}(k)^{2}=(\eta,-1)_{F}$ if $F$ is nonarchimedean and $x_{0}(k)^{2}=\operatorname{sign}(\eta)(-i)^{j} \operatorname{det}(D-i C)$ if $F=\mathbb{R}$. 
In $\S 2$ we discuss the Weil index, which is fundamental to the study of the Weil representation, and we give a new method to compute the operators belonging to $\widetilde{\mathrm{Sp}}$. In $\S 3$ we discuss splittings of the maximal compact subgroup of Sp into Mp. The main theorem is proved in $\S 4$, and as an application we construct in Proposition 10 a section $r_{+}: \mathrm{Sp} \rightarrow \widetilde{\mathrm{Sp}}$ such that the associated cocycle $c_{+}$has the following nice properties: a) $c_{+}\left(p g_{1}, g_{2}\right)=c_{+}\left(g_{1}, g_{2}\right)=$ $c_{+}\left(g_{1}, g_{2} p\right)$ for all $p=\left(\begin{array}{c}A * \\ 0 *\end{array}\right) \in \mathrm{Sp}$ with $\operatorname{det} A>0$ and all $\left.g_{1}, g_{2} \in \mathrm{Sp} ; \mathrm{b}\right) c_{+}$ has a simple formula on the standard maximal compact torus of $\mathrm{Sp}$; and c) $c_{+}$coincides with the Kubota cocycle when $m=1$. In $\S 5$ we show how to lift homomorphisms $j: \operatorname{Sp}(2 n, \mathbb{R}) \rightarrow \operatorname{Sp}(2 m, \mathbb{R})$ to the double covers of these groups.

We would like to thank the referee for many insightful comments, simplifications of arguments (for example, the proof of (4.10)), and specific references to the literature which helped to improve and shorten the paper.

\section{The Weil index.}

We first show that $\widetilde{\mathrm{Sp}}$ is equal to the commutator subgroup of $\mathrm{Mp}$ (this fact is known). By computing some commutators explicitly, we are led to consider some products of Gauss sums which Rao calls the Weil index. We will give a new proof that the Weil index defines a homomorphism of the Witt group and a new way to compute the operators in $\widetilde{\mathrm{Sp}}$. If $H$ is any group, let $(H, H)$ denote its commutator.

Lemma 1. $\widetilde{\mathrm{Sp}}$ is equal to the commutator subgroup of $M p$. If $G_{1}$ is a subgroup of $M p$ and $\pi\left(G_{1}\right)=\mathrm{Sp}$ then $G_{1}=H \widetilde{\mathrm{Sp}}=H(\mathrm{Mp}, \mathrm{Mp})$, where $H=G_{1} \cap \mathbf{T}$. Also, $\widetilde{\mathrm{Sp}}=(\widetilde{\mathrm{Sp}}, \widetilde{\mathrm{Sp}})$.

Proof. Let $G_{0}=(\mathrm{Mp}, \mathrm{Mp})$. Then $\pi \mid G_{0}$ is surjective, because $\mathrm{Sp}=(\mathrm{Sp}, \mathrm{Sp})$. Now let $G_{1}$ be any subgroup of Mp such that the restriction of $\pi$ to $G_{1}$ is surjective and let $H=G_{1} \cap \mathbf{T}$. Then $\left(G_{1}, G_{1}\right)$ contains $G_{0}$ because given any $A, B$ in Mp there are constants $t_{1}$ and $t_{2}$ in $\mathbf{T}$ such that $t_{1} A$ and $t_{2} B$ belong to $G_{1}$; thus $A B A^{-1} B^{-1}=\left(t_{1} A\right)\left(t_{2} B\right)\left(t_{1} A\right)^{-1}\left(t_{2} B\right)^{-1} \in\left(G_{1}, G_{1}\right)$. In particular, $G_{0} \subset(\widetilde{\mathrm{Sp}}, \widetilde{\mathrm{Sp}}) \subset \widetilde{\mathrm{Sp}}$. If the inclusion of $G_{0}$ in $\widetilde{\mathrm{Sp}}$ were proper, then the exact sequence (1.6) would be split. So $\widetilde{\mathrm{Sp}}=(\widetilde{\mathrm{Sp}}, \widetilde{\mathrm{Sp}})=G_{0} \subset G_{1} \subset \mathrm{Mp}$. Given any $g \in G_{1}$ there exists $a \in \mathbf{T}$ such that $a g \in \widetilde{\mathrm{Sp}}$. Since $\widetilde{\mathrm{Sp}} \subset G_{1}, a=$ $(a g) g^{-1} \in G_{1} \cap \mathbf{T}=H$. Since $g=a^{-1}(a g)$, we see $G_{1} \subset H \widetilde{\mathrm{Sp}} \subset G_{1}$,

Let $P$ be the subgroup of matrices $\left(\begin{array}{ll}A & B \\ C & D\end{array}\right) \in$ Sp such that $C=0$. Then $P$ is generated by $\underline{a}(A) \underline{n}(B)$ such that $A \in \mathrm{GL}_{m}(F)$ and $B=B^{T} \in \mathrm{M}_{m}(F)$, and the section $r_{P}: P \rightarrow \mathrm{Mp}$ given by

$$
r_{P}(\underline{a}(A) \underline{n}(B))=\mathbf{a}(A) \mathbf{n}(B)
$$


is easily seen to be a homomorphism. Since $P$ and $\left\{w_{j} \mid j=0, \ldots, m\right\}$ generate Sp ([R1, Lemma 2.14]), their lifts $\left\{r_{P}(p) \mid p \in P\right\}$ and $\mathcal{F}_{j}$, together with $\mathbf{T}$, generate $\mathrm{Mp}$. We will use this information to compute $\widetilde{\mathrm{Sp}}=(\mathrm{Mp}, \mathrm{Mp})$ and see how Gauss sums arise in the process. In the following let $\mathcal{F}=\mathcal{F}_{m}$.

Since $(\mathrm{GL}(m, F), \mathrm{GL}(m, F))=\mathrm{SL}(m, F)$ for any field $F$ (Milnor, J., Introduction to Algebraic K-Theory, Ann. of Math. Studies No. 72, p. 25 and 28$)$, it is not hard to see that $(P, P)$ is generated by $\underline{a}(A)$ such that $A \in \mathrm{SL}_{m}(F)$ and $\underline{n}(B)$ such that $B=B^{T} \in \mathrm{M}_{m}(F)$, where $I=I_{m}$. Since a and $\mathbf{n}$ are homomorphisms,

$\mathbf{a}(A) \in \widetilde{\mathrm{Sp}}$ and $\mathbf{n}(B) \in \widetilde{\mathrm{Sp}}$ for all $A \in \mathrm{SL}_{m}(F)$ and $B=B^{T} \in \mathrm{M}_{m}(F)$.

Since $\widetilde{\mathrm{Sp}}=(\mathrm{Mp}, \mathrm{Mp})$ is normal in $\mathrm{Mp}$ and $\left(\begin{array}{l}I \\ B I\end{array}\right)=w_{m} \underline{n}(-B) w_{m}^{-1}$,

$$
\mathcal{F} \mathbf{n}(-B) \mathcal{F}^{-1} \in \widetilde{\operatorname{Sp}} \cap \pi^{-1}\left(\begin{array}{l}
I \\
B I
\end{array}\right) .
$$

Now we compute the fiber in $\widetilde{\mathrm{Sp}}$ over $\underline{a}\left(B^{-1}\right) w_{m}$ when $B$ is symmetric and invertible. The Bruhat decomposition on the big cell $(\operatorname{det} C \neq 0)$ has the form

$$
\left(\begin{array}{ll}
A & B \\
C & D
\end{array}\right)=\underline{n}\left(A C^{-1}\right) \underline{a}\left(-C^{-T}\right) w_{m} \underline{n}\left(C^{-1} D\right) .
$$

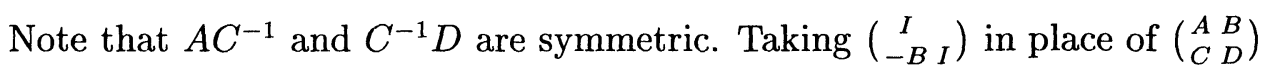
when $B$ is invertible gives

$$
\underline{n}\left(B^{-1}\right)\left(\begin{array}{c}
I \\
-B I
\end{array}\right) \underline{n}\left(B^{-1}\right)=\underline{a}\left(B^{-1}\right) w_{m} .
$$

An element of $\widetilde{\mathrm{Sp}}$ which lies in the fiber over the left side of the equation is

$$
\mathbf{n}\left(B^{-1}\right) \mathcal{F} \mathbf{n}(B) \mathcal{F}^{-1} \mathbf{n}\left(B^{-1}\right) \in \widetilde{\mathrm{Sp}}
$$

On the other hand, the fiber in Mp over the right hand side contains $\mathbf{a}\left(B^{-1}\right) \mathcal{F}$. Hence there is a constant $\gamma(B) \in \mathbf{T}$ (which depends on $\chi$ ) such that

$$
\begin{aligned}
& \gamma(B) \mathbf{a}\left(B^{-1}\right) \mathcal{F} \\
& \quad=\mathbf{n}\left(B^{-1}\right) \mathcal{F} \mathbf{n}(B) \mathcal{F}^{-1} \mathbf{n}\left(B^{-1}\right) \in \widetilde{\operatorname{Sp}} \cap \pi^{-1}\left(\left({ }_{-B} B^{-1}\right)\right)
\end{aligned}
$$


This constant is the same as $\gamma(f)$ of [W, No. 14] or the "Weil index" $\gamma_{F}(f)$ of [R1, Appendix], where $f$ is the character of second degree on $F^{m}$ given by $f(X)=\chi\left(X B X^{T} / 2\right)$.

Weil has shown that $\gamma$ is a homomorphism of the Witt group ([W, No. 25]); that is,

$$
\gamma\left(R^{T} B R\right)=\gamma(B), \quad \gamma\left(\begin{array}{ll}
B_{1} & \\
& B_{2}
\end{array}\right)=\gamma\left(B_{1}\right) \gamma\left(B_{2}\right)
$$

for all $R \in \mathrm{GL}_{m}(F)$ and all square matrices $B_{1}, B_{2}$. Here is a different proof of these facts. Take any $R \in \mathrm{GL}_{m}(F)$ such that $R^{T} B R=\operatorname{diag}\left\{b_{1}, \ldots, b_{m}\right\}$ with $b_{i} \in F^{\times}$. It will suffice to show $\gamma(B)=\prod \gamma\left(b_{i}\right)$. Let $\varphi$ be any nonzero element of $L^{2}(F)$, and define $\varphi^{\otimes m} \in L^{2}\left(F^{m}\right)$ by $\varphi^{\otimes m}(X)=\prod \varphi\left(X_{i}\right)$. Let $\Phi(X)=\varphi^{\otimes m}(X R)$. The right side of (2.5) evaluated at $\Phi$ is the function

$\Phi_{1}(X)$

$$
\begin{aligned}
& =\chi\left(X B^{-1} X^{T} / 2\right) \int_{F^{m}} \chi\left(Y B Y^{T} / 2\right)\left(\mathcal{F}^{-1} \mathbf{n}\left(B^{-1}\right) \Phi\right)(Y) \chi\left(X Y^{T}\right) d Y \\
& =\chi\left(X B^{-1} X^{T} / 2\right) \int_{F^{m}} \chi\left(Y B Y^{T} / 2\right) \int_{F^{m}} \chi\left(Z B^{-1} Z^{T} / 2\right) \Phi(Z) \chi\left((X-Z) Y^{T}\right) d Z d Y .
\end{aligned}
$$

Let $X^{\prime}=X R=\left(X_{1}^{\prime}, \ldots, X_{m}^{\prime}\right)$. Now change variables $Y \mapsto Y R^{-T}, Z \mapsto Z R$.

Then

$$
\begin{aligned}
& \Phi_{1}(X) \\
& \quad=\prod_{i=1}^{m} \chi\left(b_{i}^{-1}{X_{i}^{\prime}}^{2} / 2\right) \int_{F} \chi\left(b_{i} y^{2} / 2\right) \int_{F} \chi\left(b_{i}^{-1} z^{2} / 2\right) \varphi(z) \chi\left(\left(X_{i}^{\prime}-z\right) y\right) d z d y .
\end{aligned}
$$

On the other hand, the left side of (2.5) evaluated at $\Phi$ is

$$
\begin{aligned}
\Phi_{1}(X) & =\gamma(B)|\operatorname{det} B|_{F}^{-1 / 2} \int_{F^{m}} \Phi(Y) \chi\left(X B^{-1} Y^{T}\right) d Y \\
& =\gamma(B) \prod_{i=1}^{m}\left|b_{i}\right|_{F}^{-1 / 2} \int_{F} \varphi(y) \chi\left(b_{i}^{-1} X_{i}^{\prime} y\right) d y \\
& =\gamma(B) \prod\left|b_{i}\right|_{F}^{-1 / 2} \hat{\varphi}\left(b_{i}^{-1} X_{i}^{\prime}\right),
\end{aligned}
$$

where $\hat{\varphi}$ is the Fourier transform of $\varphi$. Comparing the two expressions for $\Phi_{1}(X)$, we find $\gamma(B)=\prod \gamma\left(b_{i}\right)$, where $\gamma(b)$ for $b \in F^{\times}$is the constant in $\mathbf{T}$ such that

$$
\begin{aligned}
& \gamma(b)|b|^{-1 / 2} \hat{\varphi}\left(b^{-1} x\right) \\
& \quad=\chi\left(b^{-1} x^{2} / 2\right) \int_{F} \chi\left(b y^{2} / 2\right) \int_{F} \chi\left(b^{-1} z^{2} / 2\right) \varphi(z) \chi((x-z) y) d z d y
\end{aligned}
$$


Note that our notation is consistent: if $m=1$ and $B=b$ then $\gamma(B)=$ $\prod_{i=1}^{1} \gamma(b)=\gamma(b)$. This proves $(2.6)$.

If $F=\mathbb{R}$ and $\chi(t)=e^{2 \pi i \lambda t}, \gamma(b)$ may be evaluated by taking $\phi(x)=e^{-\pi x^{2}}$ in formula (2.7) and applying [Ig, Ch. I, §2, Lemma 1]. The result is

$$
\gamma(b)= \begin{cases}e^{2 \pi i / 8} & \text { if } \lambda b>0 \\ e^{-2 \pi i / 8} & \text { if } \lambda b<0\end{cases}
$$

If $F$ is nonarchimedean with a discrete valuation $v$, then by [W, No. 27],

$$
\gamma(b)=\tilde{\gamma}(b) /|\tilde{\gamma}(b)|, \quad \tilde{\gamma}(b)=\sum_{\nu=-\infty}^{+\infty} \int_{v(y)=\nu} \chi\left(b y^{2} / 2\right) d y
$$

where $d y$ is an additive Haar measure. This is recognizable as a Gauss sum.

In ([W, No. 28]) it is shown that for all $a, b \in F^{\times}$,

$$
\gamma(-b)=\bar{\gamma}(b), \quad \gamma(1) \gamma(-a) \gamma(-b) \gamma(a b)=(a, b)_{F},
$$

where $(a, b)_{F}=1$ or -1 according as $a$ is or is not a norm in $F\left(b^{1 / 2}\right)$. From this it is easy to deduce that $\gamma(b)^{8}=1$, and even $\gamma(a)^{4} \gamma(b)^{4}=1$, for all $a, b \in F^{\times}$. Other formulas are gathered in the appendix of $[\mathbf{R 1}]$.

Proposition 2. For all $A \in \mathrm{GL}_{m}(F)$ and $B=B^{T} \in \mathrm{M}_{m}(F)$,

$$
(\operatorname{det} A,-1)_{F}^{1 / 2} \mathbf{a}(A) \in \widetilde{\mathrm{Sp}}, \quad \mathbf{n}(B) \in \widetilde{\mathrm{Sp}}, \quad \gamma(1)^{j} \mathcal{F}_{j} \in \widetilde{\mathrm{Sp}} .
$$

Proof. These formulas can be deduced from [R1, Def. 5.2 and Cor. A.5] or from [R2, Th. 4.1]; here we give a different proof. The first assertion when $\operatorname{det} A=1$, the second assertion in general, and the third assertion when $j=$ $m$ have already been shown (equations (2.2) and (2.5)). For arbitrary $A \in$ $\mathrm{GL}_{m}(F)$, write $A=B A_{1}$ with $B=\operatorname{diag}\left\{\operatorname{det} A, I_{m-1}\right\}$ and $A_{1} \in \mathrm{SL}_{m}(F)$. Then

$$
\frac{\gamma(\operatorname{det} A)}{\gamma(1)} \mathbf{a}(A)=\{\gamma(B) \mathbf{a}(B) \mathcal{F}\}\{\gamma(I) \mathcal{F}\}^{-1}\left\{\mathbf{a}\left(A_{1}\right)\right\}
$$

Here we have used that $\gamma(B) / \gamma(I)=\gamma(\operatorname{det} A) / \gamma(1)$ by (2.6). Each term in brackets belongs to $\widetilde{\mathrm{Sp}}$ by $(2.5)$ and $(2.2)$. Now

$$
\{\gamma(1) / \gamma(a)\}^{2}=\gamma(1)^{2} \gamma(-a)^{2}=(a,-1)_{F}
$$

by $(2.10)$, so $\gamma(\operatorname{det} A) / \gamma(1)= \pm(\operatorname{det} A,-1)_{F}^{1 / 2}$. This proves the first assertion. One can deduce the last assertion by embedding $\operatorname{Sp}(2 j, F)$ into $\operatorname{Sp}(2 m, F)\left(\left(\begin{array}{ll}a & b \\ c & d\end{array}\right) \mapsto\left(\begin{array}{ll}A & B \\ C & D\end{array}\right)\right.$, where $A=\left(\begin{array}{cc}a & \\ & I_{m-j}\end{array}\right), B=\left(\begin{array}{cc}{ }^{b} & \\ 0_{m-j}\end{array}\right), C=\left(\begin{array}{cc}{ }^{c} & \\ 0_{0_{m-j}}\end{array}\right)$, $\left.D=\left({ }^{d} I_{m-j}\right)\right)$ and applying the argument of $(2.4-5)$ to the image of $\operatorname{Sp}(2 j, F)$. 


\section{Splitting of $K$.}

In this section we recall how to obtain the splitting of a maximal compact subgroup $K$ of Sp. First let us discuss the case where $F=\mathbb{R}$. Let $U_{m}=$ $\left\{Z \in \mathrm{M}_{m}(\mathbb{C}) \mid Z \bar{Z}^{T}=I_{m}\right\}$, and define $u: U_{m} \rightarrow$ Sp by the formula

$$
u(A+i B)=\left(\begin{array}{cc}
A & B \\
-B & A
\end{array}\right) .
$$

It is well known that Sp acts on the Siegel upper half space, and by an easy calculation $u\left(U_{m}\right)$ is the stabilizer of $i I_{m}$, hence it is a maximal compact subgroup of Sp. The nontrivial additive character $\chi$ which appears in (1.2) and (1.3) has the form $\chi(t)=e^{2 \pi i \lambda t}$, where $\lambda \in \mathbb{R}^{\times}$. Define $\Phi_{0} \in L^{2}\left(\mathbb{R}^{m}\right)$ by

$$
\Phi_{0}\left(X_{1}, \ldots, X_{m}\right)=e^{-\pi|\lambda| \sum X_{j}^{2}} .
$$

Lemma 3. Let $F=\mathbb{R}$ and $K=u\left(U_{m}\right)$. There is a splitting homomorphism $\mathbf{k}: K \rightarrow \operatorname{Mp}$ such that $\mathbf{k}(k)\left(\Phi_{0}\right)=\Phi_{0}$ for all $k \in K$.

Proof. It is well known that $\pi^{-1}(K)$ stabilizes $\mathbb{C}^{\times} \Phi_{0}$; see [Ig, Ch. I, §9] or $\left[\right.$ B, Prop. 3.2(a)]. Let $\tilde{\tau} \in \mathrm{Mp}$ and $\pi(\tilde{\tau})=k \in K$. Then $\tilde{\tau} \Phi_{0}=c \Phi_{0}$, and $c \in \mathbf{T}$ since $\tilde{\tau}$ is a unitary operator. Thus one takes $\mathbf{k}(k)=c^{-1} \tilde{\tau}$.

There is a similar result in the nonarchimedean case $([\mathbf{W}$, No. 19] or [Kz, Lemma 2]). Let $F$ be a nonarchimedean field, $\mathfrak{o}_{F}$ its ring of integers, $\Lambda$ a lattice in $F^{m}$, and $\Lambda^{\prime}$ the dual lattice:

$$
\Lambda^{\prime}=\left\{x \in F^{m} \mid \chi(x \cdot y)=1 \quad \text { for all } y \in \Lambda\right\} .
$$

Let $K \subset$ Sp be the stabilizer of $\Lambda \oplus \Lambda^{\prime}$ :

$$
K=\left\{\sigma \in \operatorname{Sp} \mid(x, y) \sigma \in \Lambda \oplus \Lambda^{\prime} \quad \text { for all } x \in \Lambda, y \in \Lambda^{\prime}\right\} .
$$

Then $K$ is a maximal compact subgroup of Sp. Since $\Lambda=\mathfrak{o}_{F}^{m} \alpha$ for some $\alpha \in \mathrm{GL}_{m}(F)$, the various $K$ for different choices of $\Lambda$ are conjugate to one another. If $\Lambda=\mathfrak{o}_{F}^{m}$ and $\mathfrak{a}_{\chi}=\mathfrak{o}_{F}$ (see (1.8)) then $\Lambda^{\prime}=\mathfrak{o}_{F}^{m}$ and $K=$ $\operatorname{Sp}\left(2 m, o_{F}\right)$.

Lemma 4. Let $F$ be a nonarchimedean field such that $2 \in \mathfrak{o}_{F}^{\times}$. Let $\Phi_{0}$ be the characteristic function of $\Lambda$. There is a splitting homomorphism $\mathbf{k}: K \rightarrow \widetilde{\mathrm{Sp}}$ such that $\mathbf{k}(k) \Phi_{0}=\Phi_{0}$ for all $k \in K$.

Proof. This is proved in [Kz, Lemma 2]. (N.B. Kazhdan's article contains an error in the group law for the Heisenberg group, but the reasoning is correct in the case where $2 \in \mathfrak{o}_{F}^{\times}$.) 
Lemma 5. Let $F=\mathbb{R}$ or $F$ as in the previous lemma. If $p \in P \cap K$ then $r_{P}(p)=\mathbf{k}(p)$. Let $\widehat{P}=w_{m}^{-1} P w_{m}=\left\{\left(\begin{array}{ll}A & B \\ C & D\end{array}\right) \in \mathrm{Sp} \mid B=0\right\}$ and define $r_{\widehat{P}}: \widehat{P} \rightarrow \operatorname{Mp}$ by $r_{\widehat{P}}\left(w_{m}^{-1} p w_{m}\right)=\mathcal{F}^{-1} r_{P}(p) \mathcal{F}$. If $\hat{p} \in \widehat{P} \cap K$ then $r_{\widehat{P}}(\hat{p})=\mathbf{k}(\hat{p})$.

Proof. Suppose $p \in P \cap K$. We can write $p=\underline{n}(B) \underline{a}(A)$. From the definition of $K$ it is clear that $\operatorname{det} A$ is a unit. Write $r_{P}(p)=c \mathbf{k}(k)$, with $c \in \mathbf{T}$. Since $\Phi_{0}(0)=1$ and $\mathbf{k}(k) \Phi_{0}=\Phi_{0}$, we know $c=r_{P}(p) \Phi_{0}(0)=\mathbf{n}(B) \mathbf{a}(A) \Phi_{0}(0)=$ $|\operatorname{det} A|^{1 / 2}=1$. Next suppose $\hat{p}=w_{m}^{-1} p w_{m} \in \widehat{P} \cap K$. Let $\Phi_{0}^{\prime}=\mathcal{F} \Phi_{0}$ and $K^{\prime}=w_{m} K w_{m}^{-1}$. If $F=\mathbb{R}$ then $\Phi_{0}^{\prime}=\Phi_{0}$ and $K^{\prime}=K$. If $F$ is nonarchimedean then $\Phi_{0}^{\prime}$ is a constant multiple of the characteristic function of $\Lambda^{\prime}$ and $K^{\prime}$ is the stabilizer of $\Lambda^{\prime} \oplus \Lambda$; that is, $\Phi_{0}^{\prime}(0)^{-1} \Phi_{0}^{\prime}, K^{\prime}$ are defined like $\Phi_{0}, K$ with the roles of $\Lambda$ and $\Lambda^{\prime}$ reversed. Moreover $p \in P \cap K^{\prime}$, hence $r_{P}(p) \Phi_{0}^{\prime}=\Phi_{0}^{\prime}$. Thus $r_{\widehat{P}}(\hat{p}) \Phi_{0}=\mathcal{F}^{-1} r_{P}(p) \Phi_{0}^{\prime}=\mathcal{F}^{-1} \Phi_{0}^{\prime}=\Phi_{0}$, so that $r_{\widehat{P}}(\hat{p})=\mathbf{k}(\hat{p})$.

Lemma 6. If $F$ is nonarchimedean and $2 \in \mathfrak{o}_{F}^{\times}$then $(a, b)_{F}=1$ for all $a, b \in \mathfrak{o}_{F}^{\times}$.

Proof. This proof may be found in [W, Theorem 5]. Choose $\chi$ such that $\mathfrak{a}_{\chi}=\mathfrak{o}_{F}($ see $(1.8))$. Let $\Lambda=\mathfrak{o}_{F}^{m}$; thus $K=\operatorname{Sp}\left(2 m, \mathfrak{o}_{F}\right)$. Note that $\mathbf{k}\left(w_{m}\right)=$ $\mathcal{F}$, since $\mathcal{F} \Phi_{0}=\Phi_{0}$. Consider equation (2.5) with $m=1$ and $B=b$, where $b \in \mathfrak{o}_{F}^{\times}$. By Lemma $5,(2.5)$ says

$$
\gamma(b) \mathbf{k}\left(\underline{a}\left(b^{-1}\right)\right) \mathbf{k}\left(w_{m}\right)=\mathbf{k}\left(\underline{n}\left(b^{-1}\right)\right) \mathbf{k}\left(w_{m}\right) \mathbf{k}(\underline{n}(b)) \mathbf{k}\left(w_{m}\right)^{-1} \mathbf{k}\left(\underline{n}\left(b^{-1}\right)\right) .
$$

Since $\mathbf{k}$ is a homomorphism, $\gamma(b)=1$. Then $(a, b)_{F}=1$ by $(2.10)$.

Lemma 7. If $F$ is nonarchimedean and $2 \in \mathfrak{o}_{F}^{\times}$then $\mathbf{k}(k) \in \widetilde{\mathrm{Sp}}$ for all $k \in K$. If $F=\mathbb{R}$ then $\pm(\operatorname{det} Z)^{1 / 2} \mathbf{k}\left(u\left(Z_{\lambda}\right)\right) \in \widetilde{\mathrm{Sp}}$ for all $k \in K$, where $Z_{\lambda}=Z$ or $\bar{Z}$ according as $\lambda>0$ or $\lambda<0$.

Proof. First assume $F$ is nonarchimedean. It is well known that $K$ is generated by $K \cap P$ and $K \cap \widehat{P}$, where $\widehat{P}=w_{m}^{-1} P w_{m}$. Suppose $p=\underline{n}(B) \underline{a}(A) \in$ $P \cap K$. Then $\mathbf{k}(p)=r_{P}(p)$ by Lemma 5 . Now $\operatorname{det} A \in \mathfrak{o}_{F}^{\times}$, so $r_{P}(p) \in \widetilde{\mathrm{Sp}}$ by Lemma 6 and Prop. 2. Next suppose $\hat{p}=w_{m}^{-1} p w_{m} \in \widehat{P} \cap K$. By Lemma 5, $\mathbf{k}(\hat{p})=r_{\widehat{P}}(\hat{p})$. Now $r_{P}(p) \in \widetilde{\mathrm{Sp}}$ by the same argument as above. (Note that $p \in P \cap K^{\prime}$ in the proof of Lemma 5.) Since $\widetilde{\mathrm{Sp}}$ is normal in $\mathrm{Mp}, r_{\widehat{P}}(\hat{p}) \in \widetilde{\mathrm{Sp}}$ also.

Next assume $F=\mathbb{R}$. In [R2, Th. 4.1] a homomorphism $\tilde{\sigma} \mapsto r(\tilde{\sigma})$ from the universal cover of $\mathrm{Sp}$ into $\mathrm{Mp}$ is constructed. From the construction it is easy to see that for each $\sigma \in \mathrm{Sp}$, the set $\{r(\tilde{\sigma}) \mid \pi(\tilde{\sigma})=\sigma\}$ has cardinality two. By Lemma 1 it follows that the image of $r$ is Sp. When $\lambda=1$, the result now follows from [R2, Prop. 4.2]. Let us add a subscript $\chi$ to remind 
ourselves that the definition of $\mathbf{k}$ depends on $\chi$. From (1.7) and the definition of $\Phi_{0}$ it is not hard to deduce that $\mathbf{k}_{\chi}(u(Z))=\mathbf{k}_{\lambda \chi}\left(u\left(Z_{\lambda}\right)\right)$ for all $\lambda \in \mathbb{R}^{\times}$, $Z \in U_{m}$. The result follows.

\section{Proof of the Main Theorem.}

In this section we will prove the Main Theorem, which was stated in the introduction, and give an application. Let $F=\mathbb{R}$ or $F=$ a self-dual nonarchimedean field such that 2 is a unit in $o_{F}$. Let $\chi(t)=e^{2 \pi i t}$ if $F=\mathbb{R}$, and let $\chi(t)$ be an additive character of $F$ such that $\mathfrak{a}_{\chi}=\mathfrak{o}_{F}$ (see (1.8)) if $F$ is nonarchimedean. Let $K=u\left(U_{m}\right)$ if $F=\mathbb{R}$ (see (3.1)) and $K=\operatorname{Sp}\left(2 m, \mathfrak{o}_{F}\right)$ if $F$ is nonarchimedean. For each $k \in K$ we will define a number $x_{0}(k) \in \mathbf{T}$, which will turn out to be the same as the Gauss sum $x_{0}(k)$ that appears in the statement of the Main Theorem. Moreover $x_{0}(k)^{2}$ can easily be evaluated using results of $\S \S 2-3$.

Let us explain the definition of $x_{0}(k)$. By the Bruhat decomposition ([R1, Lemma 2.14]), every element $g \in$ Sp can be written in the form $g=p_{1} w_{j} p_{2}$ for some $p_{1}, p_{2} \in P$ and $0 \leq j \leq m$. Consider the standard section $r_{0}: \mathrm{Sp} \rightarrow \mathrm{Mp}$ given by

$$
r_{0}\left(p_{1} w_{j} p_{2}\right)=r_{p}\left(p_{1}\right) \mathcal{F}_{j} r_{P}\left(p_{2}\right)
$$

This is well-defined by virtue of [R1, Th. 3.5(3)]. Define $x_{0}(k) \in \mathbf{T}$ for $k \in K$ by the formula

$$
r_{0}(k)=x_{0}(k) \mathbf{k}(k)
$$

Suppose $y(k), y_{0}(g)$ are elements of $\mathbf{T}$ such that $y(k) \mathbf{k}(k) \in \widetilde{\mathrm{Sp}}$ and $y_{0}(g) r_{0}(g) \in$

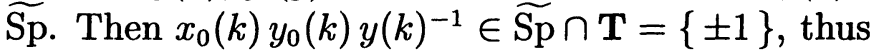

$$
x_{0}(k)^{2}=y(k)^{2} y_{0}(k)^{-2} .
$$

By virtue of Lemma 7, Prop. 2, and (2.8), we can take (for $g=\left(\begin{array}{c}A_{1} * \\ 0\end{array}\right) w_{j}\left(\begin{array}{c}A_{2} * \\ 0\end{array}\right) \in$ Sp and $k \in K$ )

$$
y(k)=\operatorname{det}\left(u^{-1}(k)\right)^{1 / 2} \quad \text { and } \quad y_{0}(g)=e^{2 \pi i j / 8}\left(\operatorname{sign}\left(\operatorname{det} A_{1} A_{2}\right)\right)^{1 / 2} \quad \text { if } \quad F=\mathbb{R},
$$

$$
y(k)=1 \quad \text { and } \quad y_{0}(g)=\left(\operatorname{det} A_{1} A_{2},-1\right)_{F}^{1 / 2} \quad \text { if } \quad F \text { is nonarchimedean. }
$$


Now we begin the computation of $x_{0}(k)$. Suppose $k=p_{1} w_{j} p_{2}$ is the Bruhat decomposition of $k$ and $p_{1}=\underline{n}\left(B_{1}\right) \underline{a}\left(A_{1}\right)$. Note that $j$ is the rank of the bottom left $m \times m$ minor of $k$. Then

$$
x_{0}(k)=\left(r_{0}(k) \Phi_{0}\right)(0)=\left|\operatorname{det} A_{1}\right|^{1 / 2} \int_{F^{j}}\left(r_{P}\left(p_{2}\right) \Phi_{0}\right)(y, 0) d y .
$$

Thus we need to calculate $A_{1}$ and $p_{2}$. This is accomplished in the next two lemmas.

Lemma 8. Given $C \in \mathrm{M}_{m}(F)$ of rank $j$, there exist $\pi_{1}, \pi_{2} \in \mathrm{SO}(m, F)$ with all entries 0,1 , or -1 such that the top left $j \times j$ minor of $\pi_{1} C \pi_{2}$ is invertible.

Proof. One can permute columns to make the first $j$ columns of $C$ linearly independent, then one can exchange rows to make the top left $j \times j$ minor of $C$ invertible. The resulting matrix is $\pi_{1}^{\prime} C \pi_{2}^{\prime}$, where $\pi_{1}^{\prime}$ and $\pi_{2}^{\prime}$ are permutation matrices. One can always arrange for an even number of row exchanges (to make $\operatorname{det} \pi_{i}^{\prime}=1$ ) unless $m=2, j=1$. When $m=2$, one could choose $\pi_{i}=I_{2}$ or $\left(\begin{array}{rr}0 & 1 \\ -1 & 0\end{array}\right)$.

Lemma 9. Suppose $g=\left(\begin{array}{ll}A & B \\ C & D\end{array}\right) \in \mathrm{Sp}(2 m, F)$, the rank of $C$ is $j$, and the top left $j \times j$ minor of $C$ is invertible. Write

$$
C=\left(\begin{array}{ll}
C_{1} & C_{2} \\
C_{3} & C_{4}
\end{array}\right) \quad D=\left(\begin{array}{cc}
D_{1} & D_{2} \\
D_{3} & D_{4}
\end{array}\right)
$$

in block form, where $C_{1}, D_{1}$ are $j \times j$ matrices and $C_{4}, D_{4}$ are $(m-j) \times(m-j)$ matrices. Then $g=p_{1} w_{j} p_{2}$, where $w_{j}=\pi\left(\mathcal{F}_{j}\right)$ is the matrix of (1.5) and $p_{1}, p_{2} \in P$ have the form

$$
\begin{aligned}
& p_{1}=\left(\begin{array}{rr}
\left(\alpha^{\prime T}\right)^{-1} & * \\
& \alpha^{\prime}
\end{array}\right), \quad \quad \alpha^{\prime}=\left(\begin{array}{ll}
-C_{1} & D_{2} \\
-C_{3} & D_{4}
\end{array}\right), \\
& p_{2}=\left(\begin{array}{cc}
I_{m} & B^{\prime} \\
& I_{m}
\end{array}\right)\left(\begin{array}{cc}
\alpha & \\
& \left(\alpha^{T}\right)^{-1}
\end{array}\right), \quad \alpha=\left(\begin{array}{cc}
I_{j} & C_{1}^{-1} C_{2} \\
& I_{m-j}
\end{array}\right), \\
& B^{\prime}=\left(\begin{array}{cc}
\beta & \\
& 0_{m-j}
\end{array}\right), \quad \beta=C_{1}^{-1}\left(D_{1}+D_{2} C_{2}{ }^{T} C_{1}{ }^{-T}\right) \in \mathrm{M}_{j}(F) .
\end{aligned}
$$

(In particular, we are asserting that $\alpha^{\prime}$ is invertible and $\beta$ is symmetric, so that $p_{1}, p_{2}$ really belong to $P$.)

Proof. First, $D C^{T}$ is symmetric since $g \in \mathrm{Sp}$, thus $D_{1} C_{1}{ }^{T}+D_{2} C_{2}{ }^{T}$ is symmetric. This implies $\beta=\beta^{T}$, so $p_{2} \in P$. Now $C \alpha^{-1}=\left(\begin{array}{l}C_{1} \\ C_{3} *\end{array}\right)$. Since 
$\operatorname{rank}\left(C \alpha^{-1}\right)=\operatorname{rank}(C)=\operatorname{rank}\left(C_{1}\right)$, the ${ }^{*}$ is really 0. Thus

$$
g p_{2}^{-1}=\left(\begin{array}{cc}
A \alpha^{-1} & * \\
\left(\begin{array}{cc}
C_{1} & 0 \\
C_{3} & 0
\end{array}\right) & D^{\prime}
\end{array}\right), \quad D^{\prime}=\left(\begin{array}{cc}
-C_{1} \beta & 0 \\
-C_{3} \beta & 0
\end{array}\right)+D \alpha^{T} .
$$

By direct calculation, $D^{\prime}=\left(\begin{array}{cc}0 & D_{2} \\ D_{3}^{\prime} & D_{4}\end{array}\right)$ for some matrix $D_{3}^{\prime}$. Since $g p_{2}^{-1} \in \mathrm{Sp}$, we know $\left(\begin{array}{ll}C_{1} & 0 \\ C_{3} & 0\end{array}\right) D^{\prime T}=\left(\begin{array}{ccc}0 & C_{1} D_{3}^{\prime T} \\ 0 & { }^{*}\end{array}\right)$ is symmetric; since $C_{1}$ is invertible, this forces

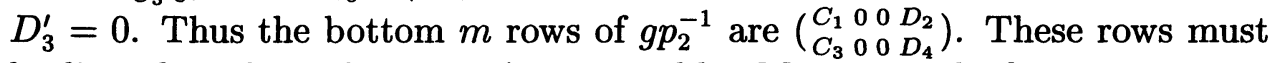
be linearly independent, so $\alpha^{\prime}$ is invertible. Moreover, the bottom $m$ rows of $\operatorname{diag}\left\{\alpha^{\prime T}, \alpha^{\prime-1}\right\} g p_{2}^{-1}$ are the same as the bottom $m$ rows of $w_{j}$. Since this matrix is symplectic, it is easy to check from the relations $A^{T} C=C^{T} A$, $B^{T} D=D^{T} B, A^{T} D-C^{T} B=I$ for all $\left(\begin{array}{ll}A & B \\ C & D\end{array}\right) \in$ Sp that the top $m$ rows of $\operatorname{diag}\left\{\alpha^{\prime T}, \alpha^{\prime-1}\right\} g p_{2}^{-1}$ must have the form

$$
\left(\begin{array}{cccc}
A_{1}^{\prime} & 0 & I_{j} & -A_{3}^{\prime T} \\
A_{3}^{\prime} & I_{m-j} & 0 & B_{4}^{\prime}
\end{array}\right)
$$

with $A_{1}^{\prime}$ and $B_{4}^{\prime}$ symmetric. (Here the matrices in the top row have height $j$, the matrices in the first and third columns have width $j$.) Thus

$$
\left(\begin{array}{ll}
I & E \\
0 & I
\end{array}\right) \operatorname{diag}\left\{\alpha^{\prime T}, \alpha^{\prime-1}\right\} g p_{2}^{-1}=w_{j}, \quad \text { where } \quad E=\left(\begin{array}{c}
A_{1}^{\prime} A_{3}^{\prime T} \\
A_{3}^{\prime}-B_{4}^{\prime}
\end{array}\right) \text {. }
$$

This completes the proof.

Proof of Main Theorem. Define $x_{0}(k)$ as in (4.2). Let $k=\left(\begin{array}{ll}A & B \\ C & D\end{array}\right) \in K-P$ and choose $\pi_{1}, \pi_{2}$ as in Lemma 8. Let $\alpha_{i}=\underline{a}\left(\pi_{i}\right)$ for $i=1,2$ and $k^{\prime}=\alpha_{1} k \alpha_{2}$. Then $x_{0}(k)=x_{0}\left(k^{\prime}\right)$ because

$$
r_{0}\left(k^{\prime}\right)=\mathbf{a}\left(\pi_{1}\right) r_{0}(k) \mathbf{a}\left(\pi_{2}\right)=\mathbf{k}\left(\alpha_{1}\right) x_{0}(k) \mathbf{k}(k) \mathbf{k}\left(\alpha_{2}\right)=x_{0}(k) \mathbf{k}\left(k^{\prime}\right)
$$

Define $C_{1}, \ldots, C_{4}$ and $D_{1}, \ldots, D_{4}$ by (1.9). Now apply Lemma 9 to the matrix $k^{\prime}$. By (4.6) and Lemma 9, the matrix $\alpha^{\prime}=\left(\begin{array}{ll}-C_{1} & D_{2} \\ -C_{3} & D_{4}\end{array}\right)$ is invertible, and in the notation of Lemma 9 ,

$$
\begin{aligned}
x_{0}\left(k^{\prime}\right) & =\left|\operatorname{det}\left(\alpha^{\prime}\right)\right|^{-1 / 2} \int_{F^{j}}\left(\mathbf{n}\left(B^{\prime}\right) \mathbf{a}(\alpha) \Phi_{0}\right)(y, 0) d y \\
& =\left|\operatorname{det}\left(\alpha^{\prime}\right)\right|^{-1 / 2} \int_{F^{j}} \chi\left(y \beta y^{T} / 2\right) \Phi_{0}\left(y, y C_{1}^{-1} C_{2}\right) d y .
\end{aligned}
$$

By changing variables $y \mapsto y C_{1}$ we obtain (1.10). Finally, $x_{0}(k)^{2}$ can be computed from (4.3), (4.4), and (4.5). 
Example. For $F=\mathbb{Q}_{p}(p \geq 3)$ and $k=\left(\begin{array}{ll}a & b \\ c & d\end{array}\right) \in \operatorname{Sp}\left(2, \mathbb{Z}_{p}\right)$, we have $x_{0}(k)=$ 1 if $c=0$, and otherwise

$$
x_{0}(k)=|c|^{1 / 2} \int_{c^{-1} \mathbb{Z}_{p}} \chi\left(d c y^{2} / 2\right) d y=|c|^{-1 / 2} \int_{\mathbb{Z}_{p}} \chi\left(d c^{-1} y^{2} / 2\right) d y .
$$

If $c$ is a unit, this integral is one. If $c$ is not a unit, then $\int_{p^{-\nu} \mathbb{Z}_{p}} \chi\left(d c^{-1} y^{2} / 2\right) d y=$ 0 for all $\nu>0$ by a standard argument in the theory of Gauss sums, so $x_{0}(k)=\gamma(d / c)$ by $(2.9)$. In summary, for $k=\left(\begin{array}{ll}a & b \\ c & d\end{array}\right) \in \operatorname{Sp}\left(2, \mathbb{Z}_{p}\right)$,

$$
x_{0}(k)= \begin{cases}\gamma(c / d) & \text { if } c \neq 0 \text { and } p \mid c \\ 1 & \text { if } c=0 \text { or } c \in \mathbb{Z}_{p}^{\times} .\end{cases}
$$

Proposition 10. Let $F=\mathbb{R}, \chi(t)=e^{2 \pi i t}$. There is a section $r_{+}: \operatorname{Sp}(2 m, \mathbb{R}) \rightarrow$ $\widetilde{\mathrm{Sp}}(2 m, \mathbb{R})$ such that

a) $r_{+}\left(p_{1} g p_{2}\right)=r_{P}\left(p_{1}\right) r_{+}(g) r_{P}\left(p_{2}\right)$ whenever $p_{1}, p_{2} \in P_{+}=\left\{\left(\begin{array}{c}A * \\ 0 *\end{array}\right) \in\right.$ $P \mid \operatorname{det}(A)>0\}$; and

b) If $k=u\left(\operatorname{diag}\left\{e^{i \theta_{1}}, \ldots, e^{i \theta_{m}}\right\}\right)\left(-\pi<\theta_{j} \leq \pi\right)$ then

$$
r_{+}(k)=e^{-\delta \pi i / 4}\left(e^{\delta \pi i / 2}\right)^{1 / 2} e^{i \theta / 2} \mathbf{k}(k),
$$

where $\theta=\sum \theta_{j}, \delta=\sum \delta_{j}$, and $\delta_{j}=-1,0,1,2$ according as $\theta_{j} \in(-\pi, 0)$, $\theta_{j}=0, \theta_{j} \in(0, \pi)$, or $\theta_{j}=\pi$. Define a cocycle $c_{+}: \operatorname{Sp} \times \operatorname{Sp} \rightarrow\{ \pm 1\}$ by the formula $r_{+}\left(g_{1}\right) r_{+}\left(g_{2}\right)=c_{+}\left(g_{1}, g_{2}\right) r_{+}\left(g_{1} g_{2}\right)$ for $g_{1}, g_{2} \in \mathrm{Sp}$. If $m=1$ then $c_{+}$ coincides with the Kubota cocycle.

Proof. Let $W_{+}=\left\{w_{j}, w_{-} w_{j} \mid j=0, \ldots, m\right\}$, where $w_{-}=\underline{a}\left({ }^{-1} I_{m-1}\right)$. It can easily be seen from the Bruhat decomposition that $\mathrm{Sp}=\sqcup_{w \in W_{+}} P_{+} w P_{+}$. Let $\varepsilon: W_{+} \rightarrow \mathrm{Mp}$ be the restriction to $W_{+}$of $k \mapsto \operatorname{det}\left(u^{-1}(k)\right)^{1 / 2} \mathbf{k}(k)$, where we choose the branch of the square root function which has argument in $(-\pi / 2, \pi / 2]$, and define $r_{+}: \mathrm{Sp} \rightarrow \widetilde{\mathrm{Sp}}$ by

$$
r_{+}\left(p_{1} w p_{2}\right)=r_{P}\left(p_{1}\right) \varepsilon(w) r_{P}\left(p_{2}\right) \quad \text { for } w \in W_{+} \text {and } p_{1}, p_{2} \in P_{+} .
$$

This section is well-defined by [R1, Theorem 3.5(3)], and it takes values in $\widetilde{\mathrm{Sp}}$ by Prop. 2 and Lemma 7. Clearly (a) is satisfied. Now we prove (b). Define $\nu: \mathrm{Sp} \rightarrow \mathbf{T}$ by $r_{+}(g)=\nu(g) r_{0}(g)$, where $r_{0}(g)$ is the standard section (4.1). Note that $\nu(w)=\left(\operatorname{det}\left(u^{-1}(w)\right)\right)^{1 / 2}$ for $w \in W_{+}$because $r_{0}(w) \Phi_{0}=\Phi_{0}$. By (4.2),

$$
r_{+}(k)=\nu(k) x_{0}(k) \mathbf{k}(k) .
$$

Thus we need to show that for $k$ as in part (b),

$$
\nu(k) x_{0}(k)=e^{-\delta \pi i / 4}\left(e^{\delta \pi i / 2}\right)^{1 / 2} e^{i \theta / 2} .
$$


If $A_{1}, A_{2} \in \mathrm{SO}_{m}(\mathbb{R})$ and $k^{\prime}=\underline{a}\left(A_{1}\right) k \underline{a}\left(A_{2}\right)$ then $\nu\left(k^{\prime}\right)=\nu(k)$ because $r_{0}$ and $r_{+}$are constant on double cosets $P_{+} w P_{+}$, and $x_{0}\left(k^{\prime}\right)=x_{0}(k)$ by (4.7). Thus the left side of (4.9) does not change if the $\theta_{j}$ are permuted or if an even number of the $\theta_{j}$ are shifted by $\pi$. The right side is also invariant under such manipulations of the $\theta_{j}$, because $\theta-\delta \pi / 2$ and $e^{\delta \pi i / 2}$ are unchanged. Thus we are reduced to the case where $\sin \theta_{\ell} \neq 0$ for $1 \leq \ell \leq j, \sin \theta_{\ell}=0$ for $\ell>j$, and $\theta_{\ell} \in[0, \pi)$ for all $\ell \geq 2$.

If $j=0$ then $k=I$ or $k=w_{-}$according as $\theta_{1}=0$ or $\theta_{1}=\pi$. If $k=I$, both sides of (4.9) are 1. If $k=w_{-}$then $\delta=2, \theta=\pi, x_{0}(k)=1$, $\nu(k)=i$, and again both sides are equal. Now consider the case $j \geq 1$. Then $\sin \theta_{1} \neq 0$, so $\delta_{1}= \pm 1, \delta_{2}=\cdots=\delta_{j}=1$, and $\delta_{\ell}=0$ for all $\ell>j$. Let $w=w_{j}$ or $w_{-} w_{j}$ according as $\delta_{1}=1$ or $\delta_{1}=-1$. Then $k \in P_{+} w P_{+}$, so $\nu(k)=\left(\delta_{1} i^{j}\right)^{1 / 2}=\left(e^{\delta \pi i / 2}\right)^{1 / 2}$. It only remains to show $x_{0}(k)=e^{-\delta \pi i / 4} e^{i \theta / 2}$. By (1.10),

$$
x_{0}(k)=\prod_{\ell=1}^{j}\left|\sin \theta_{\ell}\right|^{-1 / 2} \int_{\mathbb{R}} e^{-\pi i y^{2} \sin \theta_{\ell} \cos \theta_{\ell}} e^{-\pi y^{2} \sin ^{2} \theta_{\ell}} d y .
$$

By [Ig, Ch. I, Lemma 1], it can easily be verified that each term in the product has positive real part, and that up to a positive constant coming from the self-dual Haar measure, its square is $\left|\sin \theta_{\ell}\right|^{-1}\left(1+i \cot \theta_{\ell}\right)^{-1}=$ $e^{i \theta_{\ell}-\left(\pi i \delta_{\ell} / 2\right)}$. Since $x_{0}(k) \in \mathbf{T}$ a priori, the positive constant is one. Note that $\theta_{\ell}-\left(\pi \delta_{\ell} / 2\right) \in(-\pi / 2, \pi / 2)$ for all $\ell \leq j$, therefore $\operatorname{Re}\left(e^{i \theta_{\ell} / 2} e^{-\delta_{\ell} \pi i / 4}\right)>0$. This completes the proof of (b).

Finally we want to show that if $m=1$ then $c_{+}$coincides with the Kubota cocycle $c_{K}$ which is defined in $[\mathbf{K}]$. We recall the definition of $c_{K}$ : if $g=$ $\left(\begin{array}{ll}a & b \\ c & d\end{array}\right) \in \mathrm{SL}_{2}(\mathbb{R})$ let $x(g)=c$ or $d$ according as $c \neq 0$ or $c=0$. In terms of the Hilbert symbol which was defined after (1.9),

$$
c_{K}\left(g_{1}, g_{2}\right)=\left(x\left(g_{1}\right), x\left(g_{2}\right)\right)_{\mathbb{R}}\left(-x\left(g_{1}\right) x\left(g_{2}\right), x\left(g_{1} g_{2}\right)\right)_{\mathbb{R}} .
$$

We can reduce the proof of (c) to the case where $g_{i} \in K$ by the following argument, which was suggested by the referee. It is clear that $x(p g), x(g p)$, and $x(g)$ have the same sign if $p \in P_{+}$, thus $c_{K}\left(p g_{1}, g_{2}\right)=c_{K}\left(g_{1}, g_{2} p\right)=c_{K}\left(g_{1}, g_{2}\right)$. Moreover $c_{K}(g, p)=1=c_{K}(p, g)$ because $(x(g), 1)_{\mathbb{R}}=(-x(g), x(g))_{\mathbb{R}}=1$. Obviously $c_{+}$satisfies the same relations because of the property (a) of $r_{+}$ and because $r_{+}\left(I_{2 m}\right)=1$. Also we know the cocycle relation

$$
c(x, y) c(x y, z)=c(y, z) c(x, y z)
$$

for $c=c_{+}$or $c=c_{K}$, where $x, y, z \in \mathrm{SL}_{2}(\mathbb{R})$. For arbitrary $g_{1}$ and $g_{2}$ write $g_{1}=p_{1} k_{1}, g_{2}=p_{2} k_{2}, g_{3}=g_{1} g_{2}=p_{1} p_{3} k_{3}$ with $p_{i} \in P_{+}$and $k_{i} \in K$. Take 
$x=k_{1}, y=p_{2}, z=k_{2}$ in the cocycle relation. Then $x y=k_{1} p_{2}=p_{3} k_{3} k_{2}^{-1}$, hence

$$
c\left(g_{1}, g_{2}\right)=c\left(k_{1}, p_{2} k_{2}\right)=c\left(p_{3} k_{3} k_{2}^{-1}, k_{2}\right) c\left(k_{1}, p_{2}\right) c\left(p_{2}, k_{2}\right)^{-1}=c\left(k_{3} k_{2}^{-1}, k_{2}\right) .
$$

Thus it suffices to show $c_{+}$and $c_{K}$ coincide on $K \times K$. Let $g_{j}=u\left(e^{i \theta_{j}}\right)$ for $j=1,2,3$, where $\theta_{j} \in(-\pi, \pi]$ and $e^{i\left(\theta_{1}+\theta_{2}\right)}=e^{i \theta_{3}}$, and let $x_{j}=1$ or -1 according as $\theta_{j} \in(-\pi, 0]$ or $\theta_{j} \in(0, \pi]$. Then $c_{+}\left(k_{1}, k_{2}\right)=e^{i\left(\theta_{1}+\theta_{2}\right) / 2} / e^{i \theta_{3} / 2}$, and this is equal to -1 iff $x_{1}=x_{2}=-x_{3}$. Since $x_{j} \equiv x\left(g_{j}\right)\left(\bmod \mathbb{R}_{+}\right)$, it can easily be seen that the two cocycles agree.

\section{Embedding $\widetilde{\operatorname{Sp}}(2 n, \mathbb{R})$ into $\widetilde{\operatorname{Sp}}(2 m, \mathbb{R})$.}

In this section let $F=\mathbb{R}, \chi(t)=e^{2 \pi i t}$. Let $P_{+}=\left\{\left(\begin{array}{cc}A & B \\ 0 & D\end{array}\right) \in \operatorname{Sp} \mid \operatorname{det} A>0\right\}$. We will prove the following proposition.

Proposition 11. Consider a continuous homomorphism $j: \operatorname{Sp}(2 n, \mathbb{R}) \rightarrow$ $\mathrm{Sp}(2 m, \mathbb{R})$ such that $j\left(P_{+}^{(n)}\right) \subset P_{+}^{(m)}$ and $j\left(K^{(n)}\right) \subset K^{(m)}$, where for clarity we have superscripted our symbols with the degree of the underlying symplectic space. Let $\psi=\operatorname{det} \circ u^{-1}: K \rightarrow \mathbf{T}$. There is an integer $N$ such that

$$
\left(\psi^{(m)} \circ j\right)(k)=\left(\psi^{(n)}(k)\right)^{N} \quad \text { for } k \in K^{(n)} .
$$

Define $\tilde{j}: \operatorname{Mp}\left(\mathbb{R}^{n}\right) \rightarrow \operatorname{Mp}\left(\mathbb{R}^{m}\right)$ by

$$
\tilde{j}\left(\zeta r_{P}(p) \mathbf{k}(k)\right)=\zeta^{N} r_{P}(j(p)) \mathbf{k}(j(k)) \quad \text { for } \zeta \in \mathbf{T}, p \in P_{+}^{(n)}, k \in K^{(n)} \text {. }
$$

Then $\tilde{j}$ is a continuous homomorphism, $\pi \circ \tilde{j}=j \circ \pi$, and $\left.\tilde{j}\right|_{\tilde{\mathrm{Sp}}(2 n, \mathbb{R})}$ takes values in $\widetilde{\mathrm{Sp}}(2 m, \mathbb{R})$. Moreover, $\left.\tilde{j}\right|_{\widetilde{\mathrm{Sp}}}$ is the only continuous function from $\widetilde{\mathrm{Sp}}(2 n, \mathbb{R})$ into $\widetilde{\mathrm{Sp}}(2 m, \mathbb{R})$ such that $\pi \circ \tilde{j}=j \circ \pi$ and $\tilde{j}(1)=1$.

If one takes $n=1$ and $j\left(\left(\begin{array}{cc}\alpha \beta & \beta\end{array}\right)\right)=\left(\begin{array}{cc}\alpha I & \beta T \\ \gamma T^{-1} & \delta I\end{array}\right)$, where $T$ is a symmetric invertible $m \times m$ real matrix, and if one identifies $\widetilde{\operatorname{Sp}}(2, \mathbb{R})$ with $\operatorname{Sp} \times\{ \pm 1\}$ via the bijection $\delta r_{+}(g) \mapsto(g, \delta)$ for $r_{+}$as given in Prop. 10 and $\delta \in\{ \pm 1\}$, . then the formula (6.2) is a concise statement of [RS, Th. 1.2].

Proof. Let $\tilde{j}$ be as in (5.2), $\widetilde{K}=\widetilde{\mathrm{Sp}} \cap \pi^{-1}(K)=\left\{ \pm \psi(k)^{1 / 2} \mathbf{k}(k) \mid k \in K\right\}$ (by Lemma 7). If $\zeta \mathbf{k}(k) \in \widetilde{K}^{(n)}$ then $\tilde{j}(\zeta \mathbf{k}(k))=\zeta^{N} \mathbf{k}(j(k)) \in \widetilde{\operatorname{Sp}}(2 m, F)$ by (5.1). (An integer $N$ exists as in (5.1) because any element of $\operatorname{Hom}\left(U_{n}, \mathbf{T}\right)$ 
is a power of determinant.) It is clear that $\tilde{j}$ is well-defined, continuous, and $\pi \circ \tilde{j}=j \circ \pi$, thus we have a commutative diagram

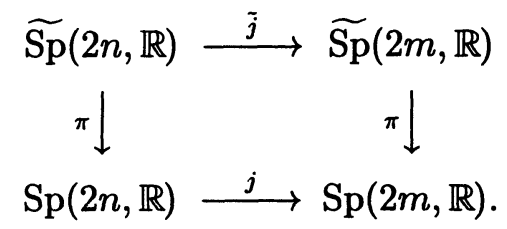

We still need to show $\tilde{j}$ is a homomorphism. Let

$$
U=\left\{p k \in \operatorname{Sp} \mid p \in P_{+}, \arg \psi(k) \in[-\pi / 2, \pi / 2]\right\}
$$

and $\phi: U \rightarrow \widetilde{\mathrm{Sp}}(2 n, \mathbb{R})$ the homeomorphism: $\phi(p k)=\psi(k)^{1 / 2} r_{P}(p) \mathbf{k}(k)$ (where $\left.\arg \psi(k)^{1 / 2} \in[-\pi / 4, \pi / 4]\right)$. Since $\phi(U)$ generates $\widetilde{\operatorname{Sp}}(2 n, \mathbb{R})$, it will suffice to show

$$
\tilde{j}(\tilde{u} \tilde{g})=\tilde{j}(\tilde{u}) \tilde{j}(\tilde{g})
$$

for all $\tilde{u}=\phi(u) \in \phi(U), \tilde{g} \in \widetilde{\mathrm{Sp}}(2 n, \mathbb{R})$. Both sides lie in the fiber of $\widetilde{\mathrm{Sp}}(2 m, \mathbb{R})$ over the point $j(u) j(g) \in \mathrm{Sp}(2 m, \mathbb{R})(g=\pi(\tilde{g}))$, so the equality holds up to \pm 1 . Let $F: U \rightarrow\{ \pm 1\}, F(u)=\tilde{j}(\tilde{u} \tilde{g}) \tilde{j}(\tilde{g})^{-1} \tilde{j}(\tilde{u})^{-1}$, where $\tilde{g} \in \widetilde{\mathrm{Sp}}$ is fixed. Then $F$ is continuous and $F\left(I_{2 m}\right)=1$. Since $U$ is connected, $F$ is identically one. This proves $\tilde{j}$ is a homomorphism. The characterization of $\tilde{j}$ as the only continuous lift of $j$ taking 1 to 1 follows from an elementary fact about covering projections ([Sp, Ch. 2, Sec. 2, Th. 2]).

\section{References}

[B] A. Bluher, The theta correspondence over $\mathbb{R}$, preprint.

[Ig] J.I. Igusa, Theta Functions, Springer-Verlag (1972), Berlin-New York.

[Kz] D. Kazhdan, Some applications of the Weil representation, Jour. d'Analyse Math., 32 (1977), 235-248.

[K] T. Kubota, Topological covering of SL(2) over a local field, J. Math. Soc. Japan, 19(1) (1967), 114-121.

[RS] S. Rallis and G. Schiffmann, Weil representation I, Intertwining distributions and discrete spectrum, Memoirs of AMS No. 231, 1980.

[R1] R.R. Rao, On some explicit formulas in the theory of the Weil representation, Pacific J. Math., 157(2) (1993), 335-371.

[R2] - The Maslov index on the simply connected covering group of the metaplectic representation, J. of Func. Anal., 107 (1992), 211-233.

[Sp] E.H. Spanier, Algebraic Topology, McGraw-Hill (1966), New Delhi. 
[W] A. Weil, Sur certains groupes d'operateurs unitaires, Acta Math., 11 (1964), 143211.

Received September 14, 1993 and revised May 9, 1994.

National Security Agency

P.O. Box 252

SimpsonVILle, MD 21150

E-mail address: bluher@afterlife.ncsc.mil 

Peng Lin and Richard Rochberg, Trace ideal criteria for Toeplitz and Hankel operators on the weighted Bergman spaces with exponential type

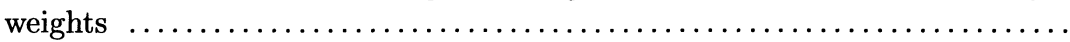

Donald E. Marshall and Arne Stray, Interpolating Blaschke products . .

Kathy D. Merrill and Lynne $\mathbf{H}$. Walling, On quadratic reciprocity over

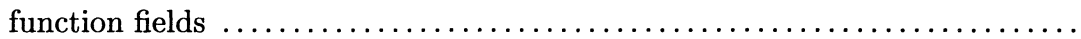

Takahiko Nakazi and Masahiro Yamada, $\left(A_{2}\right)$-conditions and Carleson

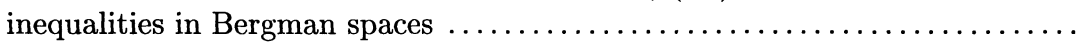

C. Ott, A note on a paper of E. Boasso and A. Larotonda ..............

Victor Patrangenaru, Classifying 3 and 4 dimensional homogeneous Riemannian manifolds by Cartan triples

Carlo Pensavalle and Tim Steger, Tensor products with anisotropic principal series representations of free groups

Ying Shen, On Ricci deformation of a Riemannian metric on manifold with boundary

Albert Jeu-Liang Sheu, The Weyl quantization of Poisson $S U(2) \ldots \ldots$

Alexandra Shlapentokh, Polynomials with a given discriminant over fields of algebraic functions of positive characteristic

Eric Stade and D.I. Wallace, Weyl's law for $S L(3, \mathbb{Z}) \backslash S L(3, \mathbb{R}) / S O(3, \mathbb{R})$

Christopher W. Stark, Resolutions modeled on ternary trees ........ 557

Per Tomter, Minimal hyperspheres in two-point homogeneous spaces .....

Jun Tomiyama, Topological Full groups and structure of normalizers in

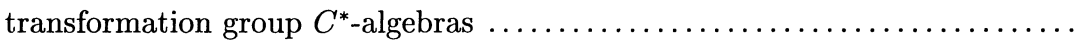

Nik Weaver, Subalgebras of little Lipschitz algebras 


\section{PACIFIC JOURNAL OF MATHEMATICS}

\section{Volume $173 \quad$ No. $2 \quad$ April 1996}

A mean value inequality with applications to Bergman space operators

PATRICK ROBERT AHERn and ZELJKO CUCKOVIC

$H^{p}$-estimates of holomorphic division formulas

MATS ANDERSSON and HASSE CARLSSON

Group structure and maximal division for cubic recursions with a double root

Christian JeAn-Claude Ballot

The Weil representation and Gauss sums

ANTONiA WiLson BLUHER

Duality for the quantum $E(2)$ group

ALFONS VAN DAELE and S. L. WORONOWICZ

Cohomology complex projective space with degree one codimension-two fixed submanifolds 387

KARL HEINZ DOVERMANN and ROBERT D. LITTLE

On the mapping intersection problem

ALEXANDER DRANISHNIKOV

From the $L^{1}$ norms of the complex heat kernels to a Hörmander multiplier theorem for

sub-Laplacians on nilpotent Lie groups

\section{XUAN THINH DUONG}

Isoperimetric inequalities for automorphism groups of free groups

Allen E. Hatcher and Karen Vogtmann

Approximation by normal elements with finite spectra in $C^{*}$-algebras of real rank zero

HUAXIN LIN

Interpolating Blaschke products

DonALD EdDY MARShall and ARNE STRAY

Interpolating Blaschke products generate $H^{\infty}$

JOHN BRADY GARNETT and ARTUR NICOLAU

Classifying 3- and 4-dimensional homogeneous Riemannian manifolds by Cartan triples

VICTOR PATRANGENARU

Polynomials with a given discriminant over fields of algebraic functions of positive

characteristic

ALEXANDRA SHLAPENTOKH

Resolutions modeled on ternary trees

CHRISTOPHER W. STARK

Topological full groups and structure of normalizers in transformation group $C^{*}$-algebras 